\title{
Resolving the Magnetic Asymmetry of the Inner Space in Self-assembled Dimeric Capsules Based on Tetraurea- calix[4]pyrrole Components
}

\author{
Mónica Espelta, Gemma Aragaya, and Pablo Ballester ${ }^{\star a b}$
}

\begin{abstract}
The encapsulation of $N, N, N^{\prime}, N^{\prime}$-tetramethyl-1,5-pentanediamine- $N, N^{\prime}$-dioxide 2 in a non-chiral capsular assembly formed by dimerization of tetraurea-calix[4]pyrrole 1a produced the observation of the $N$-methyl groups of the encapsulated guest as two separated singlets resonating highly upfield in the ${ }^{1} \mathrm{H}$ NMR spectrum. In order to clarify the origin of the observed signal splitting we assembled and studied a series of structurally related dimeric capsules. We used the tetraurea-calix[4]pyrrole 1a, the enantiomerically pure tetraurea-calix[4] pyrrole $R-\mathbf{1 b}$ and the tetraurea-bisloop calix[4]pyrrole 1c as components of the produced assemblies. The ${ }^{1} \mathrm{H}$ NMR spectra of the assembled encapsulation complexes with bis- $N$-oxide 2 evidenced diverse splitting patterns of the $N$-methyl groups. In addition, 2D EXSY/ROESY NMR experiments revealed the existence of chemical exchange processes involving the separated methyl signals of the encapsulated guest. The capsular assemblies were mainly stabilized by a belt of eight head-to-tail hydrogen-bonded urea groups. The interconversion between the two senses of rotation of the unidirectionally oriented urea groups was slow on the ${ }^{1} \mathrm{H}$ NMR timescale. These characteristics determined the appearance of a new asymmetry element (supramolecular conformational chirality) in the assemblies that accounted for some of the magnetic asymmetries featured by the capsule's inner space. The racemization of the supramolecular chirality element was fast on the EXSY timescale and produced the chemical exchange processes detected for the encapsulation complexes.
\end{abstract}

Keywords: Calix[4]pyrrole $\cdot$ Magnetic asymmetry $\cdot$ Molecular encapsulation $\cdot$ Supramolecular chirality

\section{Introduction}

Chiral supramolecular structures play a pivotal role in nature, i.e. DNA double helix, ribozymes, ribosomes etc. and in the development of synthetic molecular aggregates with novel properties and functions. ${ }^{[1,2]}$ Supramolecular chirality emerges from the assembly of a defined number of molecular components, being identical or not, into chiral architectures that are stabilized by weak intermolecular interactions. ${ }^{[3-5]}$ Interestingly, the reversible intermolecular interactions used to maintain together the assemblies' molecular components, usually hydrogen bonds or coordination bonds, may generate by themselves additional structural chiral elements. For example, the efficient establishment of arrays of intermolecular hydrogen bonds might induce the assem-

${ }^{*}$ Correspondence: Prof. P. Ballester ${ }^{a b}$

E-Mail: pballester@iciq.es

alnstitute of Chemical Research of Catalonia (ICIQ)

The Barcelona Institute of Science and Technology

Avgda. Països Catalans 16

43007 Tarragona, Spain

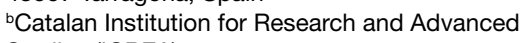

Studies (ICREA)

Passeig Lluís Companys, 23

08018 Barcelona, Spain bly of the components in conformations that are inherently chiral, ${ }^{[6,7]}$ while the use of metal-ligand interactions could generate the emergence of metal-based stereogenic centers..$^{[8]}$ In general, synthetic ${ }^{[9]}$ and biological[10,11] supramolecular systems displaying supramolecular chirality rely on the presence of stereogenic centers (chirality centers) installed in at least one of the assembled molecular units. In the same vein, axial chirality has also been demonstrated to be effective in yielding chiral synthetic assembly processes. In other words, the diastereoselective synthesis of chiral assemblies demands the use of chiral components that do not easily racemize. In addition, the point or center-chirality of the molecular unit/s must be efficiently transferred during the assembly of the supramolecular aggregate. ${ }^{[12]}$

The dimerization of chiral and achiral molecular objects constitutes paradigmatic examples to highlight the possible outcomes of self-assembly processes from the viewpoints of self-sorting and supramolecular chirality (Fig. 1 and Fig. 2).

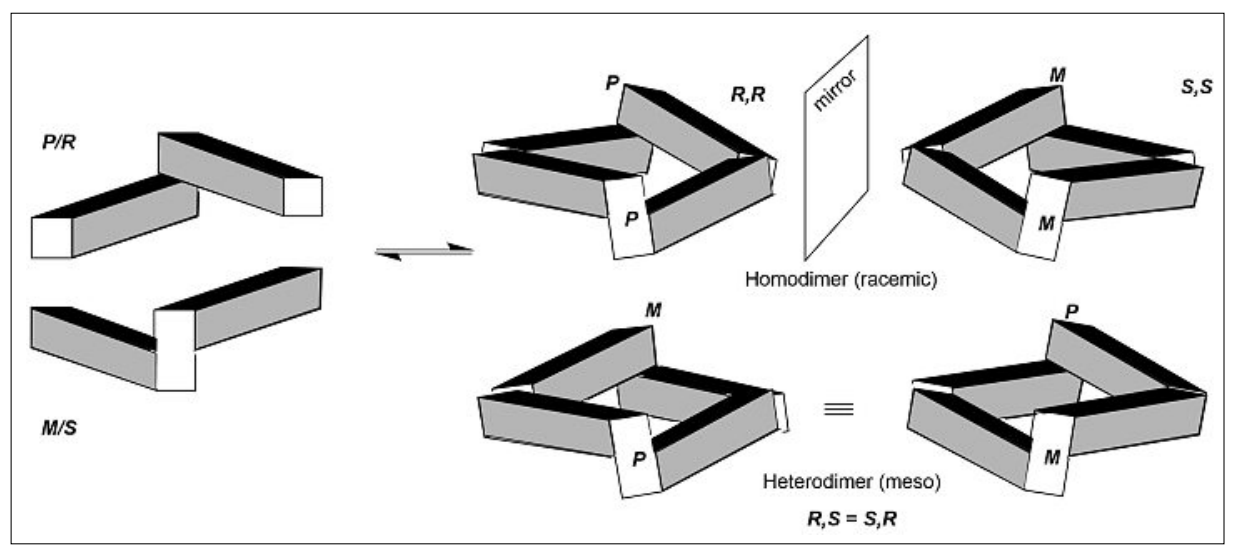

Fig. 1. Equilibria established in the reversible dimerization process of a chiral racemic monomer into dimeric aggregates. The putative appearance of additional elements of asymmetry in the dimers has not been considered for simplicity. 


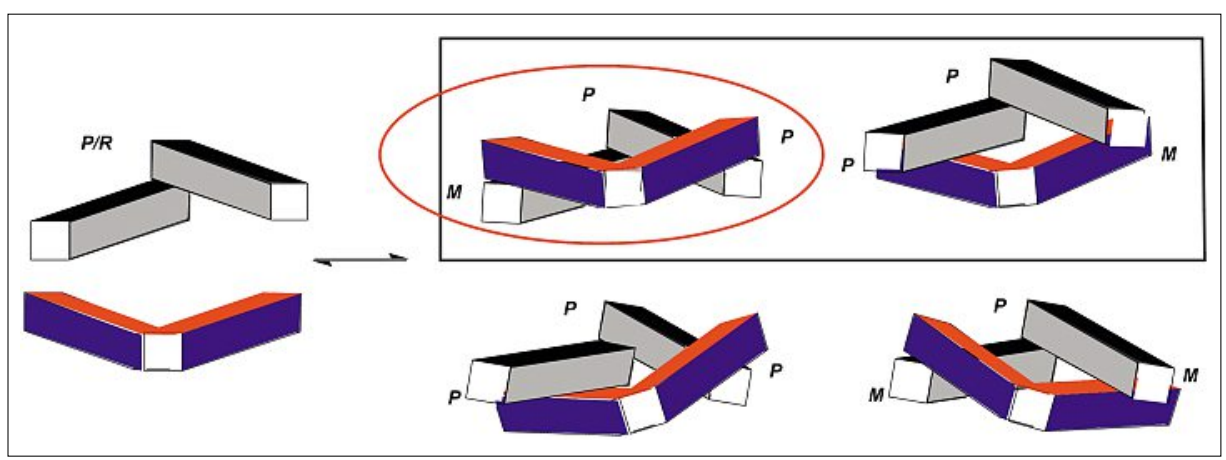

Fig. 2. Exclusive assembly of four chiral heterodimers (social-self sorting) in the dimerization of a chiral monomer with a non-chiral counterpart. To account for the formation of four chiral diastereomers we considered the appearance of two elements of asymmetry (M/P) during the dimer assembly. The plausible existence of a structurally favored relationship between the two chiral elements (identical or inverse) reduces the number of possible diastereomers to just two (black rectangle) or even to only one if the chirality point of the chiral monomer is effectively transferred to the supramolecular chirality of the dimer (red ellipsoid). The black rectangle includes the two diastereomers with complementary asymmetry elements. The detection of these two diastereomers using ${ }^{1} \mathrm{H}$ NMR spectroscopy requires that their interconversion caused by the inversion of the chiral elements resulting from their assembly (racemization) is slow on the chemical shift timescale.

To start with, the statistical self-sorting dimerization process of a chiral racemic compound is expected to produce a mixture of homodimers and heterodimer in a 1:1:2 ratio, respectively (Fig. 1). On the one hand, the exclusive or preferential formation of the homodimers will qualify the assembly process as narcissistic (selfrecognition) and diastereoselective with respect to its self-sorting properties. ${ }^{[13]}$ On the other hand, the quantitative or favored dimerization of the chiral racemic component into the heterodimer $(R, S)$ also corresponds to a self-sorting diastereoselective process, normally referred as social selfassembly (self-discrimination). ${ }^{[14,15]}$

From the supramolecular chirality point of view, the homodimers are chiral assemblies and will be produced as a racemic mixture ( $R, R$ and $S, S$, Fig. 1) while the heterodimer is meso and non-chiral $(R, S)$. The exclusive assembly of one of the enantiomeric homodimers requires the use of the enantiomerically pure monomer.

Based on the above considerations, the social self-assembly dimerization of a chiral monomer with a non-chiral counterpart will induce the formation of a chiral heterodimer as a single enantiomer. However, if two elements of chirality emerge from the assembly process up to four chiral diastereomers might be expected (Fig. 2). Due to structural constrains, the newly emerged chiral elements may have a fixed relationship (same or inverse absolute configurations), which in combination with an efficient transfer of the point-chirality might result in the exclusive formation of one of the four possible diastereomers. Such assembly process displays exquisite levels of diastereoselectivity from the viewpoints of self-sorting and supramolecular chirality.

Chiral assemblies featuring capsular topology and displaying one or multiple sizeable encapsulated molecular guests are well-known. ${ }^{[16,17]}$ In particular, the hydrogen-bonding driven dimerization of chiral and concave-shaped calix[4]arene ${ }^{[18]}$ and resorcin[4]arene ${ }^{[19]}$ derivatives, as well as their social self-assembly with nonchiral counterparts produced chiral capsular aggregates that were kinetically and thermodynamically stable. Unfortunately, the obtained chiral assemblies displayed low levels of molecular recognition for the encapsulation of chiral substrates.[20] This limitation resulted from the difficulty of transferring the point-chirality of the molecular components into their spherical or cylindrical chiral inner cavities. Nevertheless, the magnetic asymmetry present in their chiral inner spaces was easily revealed using NMR spectroscopy. Owing to the chiral nature of the assemblies, some of the protons of a non-chiral encapsulated guest became diastereotopic and resonated as separated signals in the ${ }^{1} \mathrm{H}$ NMR spectra of the corresponding encapsulation complexes. Using chiral dimeric hydrogen-bonded assemblies derived from resorcin[4]arene scaffolds, Amaya and Rebek studied and dissected the steric and magnetic asymmetry effects $^{[21]}$ caused to the encapsulated guests by the stereogenic carbon atoms located at different distances from the inner cavity. ${ }^{[22]}$

The study of the magnetic asymmetry displayed by the inner-spaces of chiral hydrogen bonded supramolecular assemblies, as well as a deeper understanding of their chiral elements (structural, stereogenic centers, axial chirality, etc.) that are responsible for the former, constitute topics of current research interest.

Some time ago, we introduced the non-chiral tetraurea-calix[4]pyrrole $\mathbf{1 a}^{[23]}$ and its enantiopure chiral version $\mathbf{1 b}$ (Fig. 3). ${ }^{[24]}$ We showed that both tetraureas reversibly dimerized forming a cyclic array of 16 hydrogen-bonds and encapsulating one molecule of 4,4'-bipyridine $N, N^{\prime}$ dioxide 3 (Fig. 4). The resulting capsular assemblies constituted a rare example of a molecular container with polar interior able to encapsulate polar guests in an ordered fashion.

More recent studies revealed that the encapsulation of a series of alkyl bis-dimethyl- $N, N$ '-dioxide guests in the mesocapsule 1a•1a produced the observation of their methyl protons as two separated singlets that resonated highly upfield shifted. ${ }^{[25]}$ We undertook the present work to clarify the origin of the observed splitting of the $N$-methyl groups for the encapsulated $\alpha, \omega$-alkanediamine $N, N, N^{\prime} N^{\prime}$ dimethyl- $N, N^{\prime}$-dioxides. In an effort to widen the study, we decided to investigate three tetraurea-calix[4]pyrroles 1a-c as capsular precursors (Fig. 3). Tetraurea 1a lacks stereogenic carbon atoms and its dimerization produced a meso capsular assembly 1a•1a (vide infra).

Tetraurea $R \mathbf{- 1 b}$ is chiral and possesses four stereogenic benzylic carbon atoms with the same absolute configuration. Its dimerization yielded the enantiopure chiral capsule $R-\mathbf{1 b} \bullet R-\mathbf{1 b}$. The dimerization process of equimolar amounts of non-chiral 1a and enantiopure $R$-1b took place with high levels of diastereoselective self-sorting and supramolecular chirality affording exclusively the heterodimeric capsular aggregate $\mathbf{1 a} \bullet R-\mathbf{1 b}$. Finally, equimolar amounts of non-chiral tetraurea 1a and non-chiral bisloop tetraurea 1c assembled almost exclusively into the heterodimeric capsule 1a•1c. All the studied capsules displayed an element of supramolecular chirality that derived from the unidirectional orientation of their urea groups. This unidirectional sense of rotation of the ureas forced the two halves of the capsule to adopt opposite cyclochiral conformations (designated as $P$ for the clockwise and $M$ for the counterclockwise sense of rotation (Fig. 4). The sense of rotation of the urea groups was kinetically stable on the ${ }^{1} \mathrm{H}$ NMR chemical shift timescale. Thus, depending on the chiral relationship that existed between the two halves (enantiomeric or diastereomeric), their intrinsic chiral nature and the symmetry properties of the assemblies, different forms and levels of supramolecular chirality emerged and could be revealed from the careful analysis of their ${ }^{1} \mathrm{H}$ NMR spectra. We selected the $N, N, N^{\prime}, N^{\prime}-$ tetramethyl-1,5-pentanediamine- $N, N$ 'dioxide $\mathbf{2}$ as template in all the dimerization studies (Fig. 2). The encapsulated bis$\mathrm{N}$-oxide 2 folds into a conformation that nicely complemented both the volume and the functionality of the inner space of all 


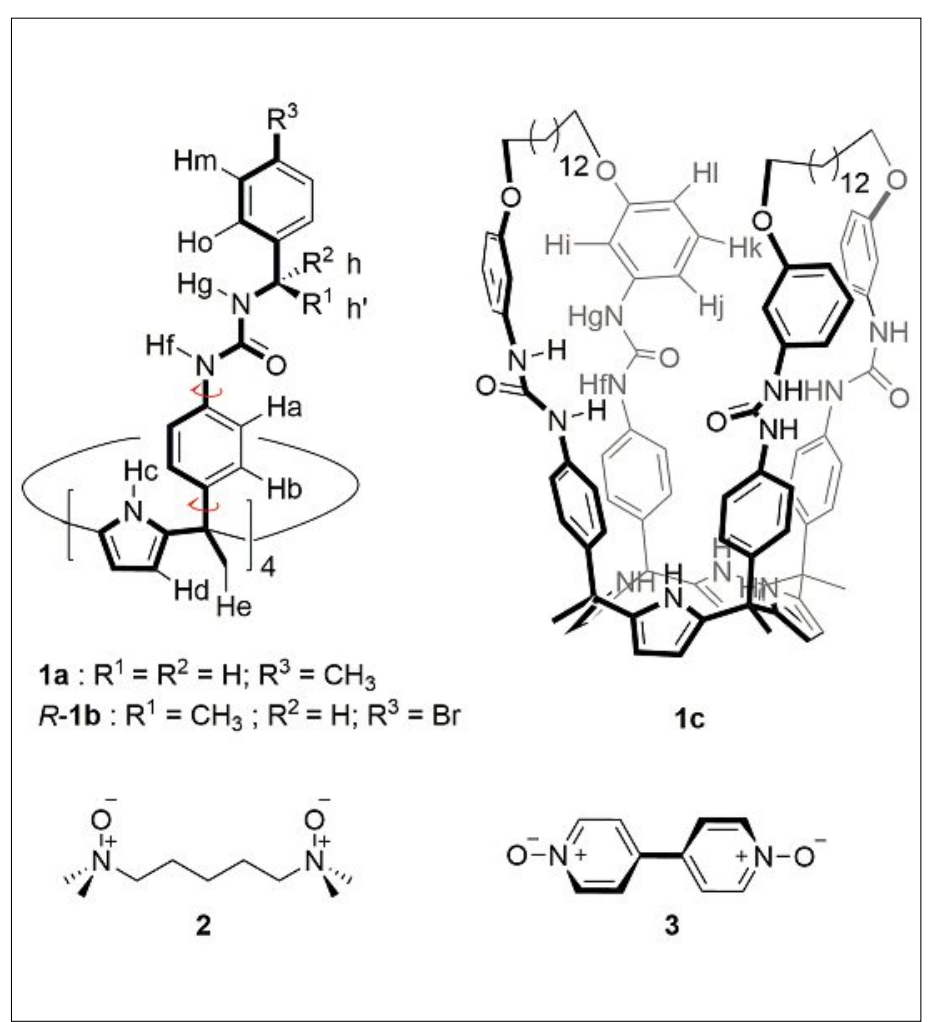

Fig. 3. Structures of the tetraurea-calix[4]pyrroles and bis- $N$-oxide guests used in this work and in previous studies described in literature.

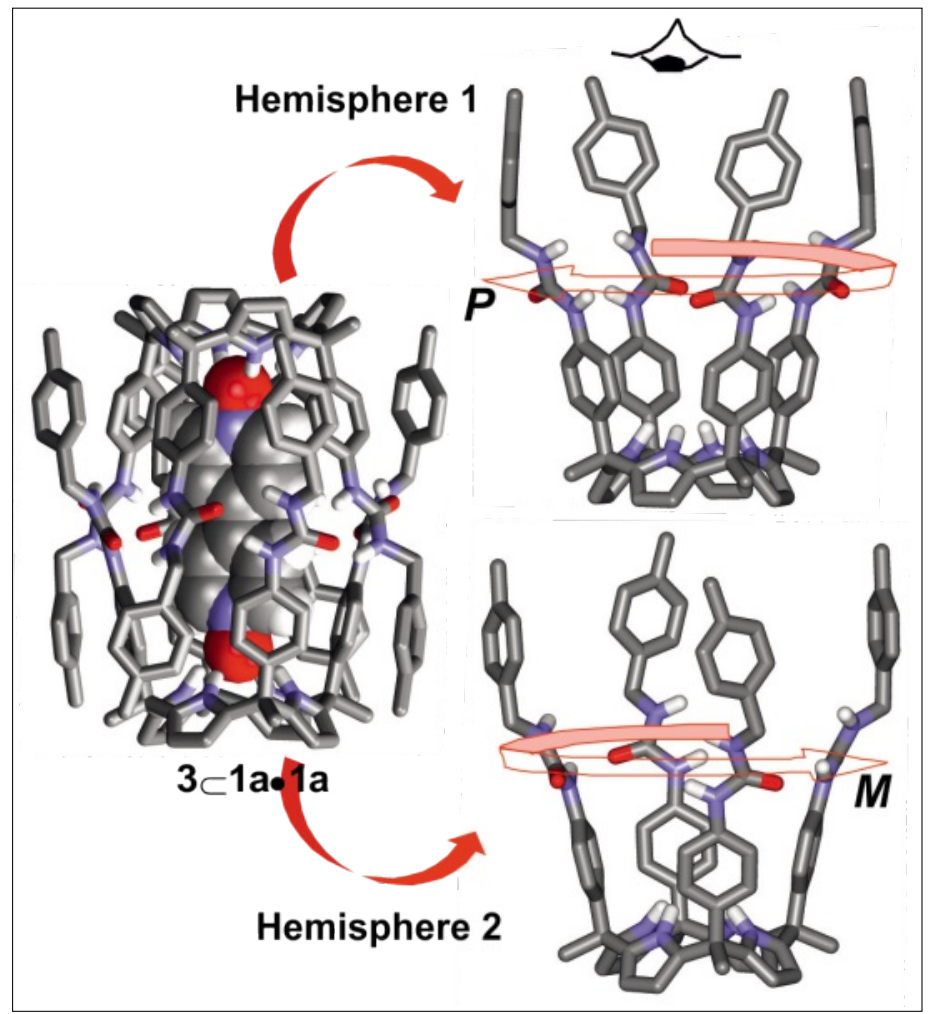

Fig. 4. Left) Energy minimized MM3 structure of the $\mathbf{3} \subset \mathbf{1 a} \mathbf{1} \mathbf{1 a}$ encapsulation complex. Right) The two calix[4]pyrrole units of the assembly (hemispheres) are shown separately to highlight the opposite and complementary sense of rotation of their urea groups. The two hemispheres are conformationally and inherently chiral cycloenantiomers. the studied dimeric capsular assemblies. ${ }^{[25]}$ The magnetic asymmetry featured by the inner-space of both chiral and non-chiral capsular assemblies caused diverse and characteristic splitting patterns of the methyl groups of the encapsulated $\mathrm{N}$-oxide 2. The observed splitting patterns were rationalized on the basis of the symmetry and the supramolecular chirality properties of the assembled capsules. Furthermore, EXSY/ROESY experiments performed in the series of encapsulation complexes evidenced distinctive chemical exchange processes involving the methyl proton signals of encapsulated 2 . These processes derived from the interconversion between the two senses of rotation of urea groups that was slow on the ${ }^{1} \mathrm{H}$ NMR timescale but fast on the EXSY/ROESY timescale.

\section{Results and Discussion}

\section{Magnetic Asymmetries in the Inner Space of Homodimers: Non-chiral Homodimeric Assembly 1a・1a and Enantiopure Homodimeric Assembly R-1b•R-1b.}

As commented above, we described the dimerization process of tetraurea-calix[4] pyrrole 1a in $\mathrm{CDCl}_{3}$ solution induced by encapsulation of one molecule of $N, N^{\prime}$ dioxide 2 (Fig. 3). [25] The resulting ho-

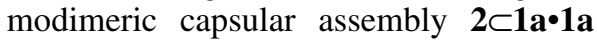

(Fig. 5) is not chiral, however the methyl protons of the encapsulated $N$-oxide $\mathbf{2}$ split into two singlets that resonate highly upfield shifted. What can be the origin of the magnetic asymmetry present in the inner cavity of a non-chiral homodimer $\mathbf{1 a} \cdot \mathbf{1 a}$ ? The dimeric assembly $\mathbf{1 a} \cdot \mathbf{1 a}$ is stabilized by the head-to-tail unidirectional orientation of their eight urea groups creating a seam of 16 hydrogen bonds. This peculiar hydrogen bonding arrangement forces the four urea groups in each one of the two tetraurea-calix[4]pyrrole monomers, 1a, to display a complementary sense of rotation when observed from a position above their cavities (Fig. 4). This is a general property exhibited by all the assembled dimeric capsules studied in this work. To comply with the above requirement the tetraureacalix[4]pyrrole monomers 1a assemble in the dimer as conformationally cyclochiral enantiomeric compounds, which we designate as $P$ and $M$ (Fig. 5a). Each hemisphere in the $\mathbf{1 a} \cdot \mathbf{1 a}$ capsule is chiral and features $C_{4}$ symmetry, however the overall dimeric assembly $\mathbf{1 a} \bullet \mathbf{1 a}$, consisting of two cyclochiral enantiomeric halves, is meso and possesses $S_{8}$ symmetry. The $C_{4}$ symmetry of the halves indicated that $\mathbf{2}$ was experiencing a fast spinning process on the chemical shift timescale.

The inclusion of the alkyl-dimethyl- $N$ oxide knobs of $\mathbf{2}$ in the deep aromatic cavity of 1a was driven by the formation of four hydrogen bonds between the oxygen atom of the $\mathrm{N}$-oxide and the pyrrole NHs. The inclusion in the aromatic cavity is evidenced by the large upfield shift experienced by the signals of the encapsulated methyl protons $(\Delta \delta \sim 2.5 \mathrm{ppm})$. The inclusion process of the alkyl-dimethyl- $N$-oxide residue in a chiral half of the $\mathbf{1 a} \cdot \mathbf{1 a}$ dimer induced the two methyl groups of the same $\mathrm{N}$-oxide knob to become diastereotopic and resonate as separated signals (Fig. 6a). The other chiral half of the $\mathbf{1 a} \cdot \mathbf{1 a}$ capsule had an enantiomeric relationship with the former and consequently all their proton signals must have identical chemical shift values. The observation of separated signals for the diastereotopic methyl groups of the encapsulated $\mathbf{2}$ also indicated that the unidirectional orientation of the eight urea groups in the $\mathbf{1 a} \cdot \mathbf{1 a}$ capsular assembly was kinetically stable on ${ }^{1} \mathrm{H}$ NMR chemical shift timescale. Otherwise the $\mathbf{1 a} \cdot \mathbf{1 a}$ capsular assembly and its two hemispheres will display $C_{4 \mathrm{v}}$ symmetry and they would not exist in solution as conformationally cycloenantiomers.

The ${ }^{1} \mathrm{H}$ NMR spectrum of $\mathbf{2} \subset \mathbf{1 a} \cdot \mathbf{1 a}$ in $\mathrm{CDCl}_{3}$ solution also reflected the chiral nature of its two hemispheres and the kinetic stability of the unidirectional orientation of the urea belt through the observation of two sets of separated signals for the ortho and meta aromatic protons with respect to the urea groups in the meso-phenyl sub- 


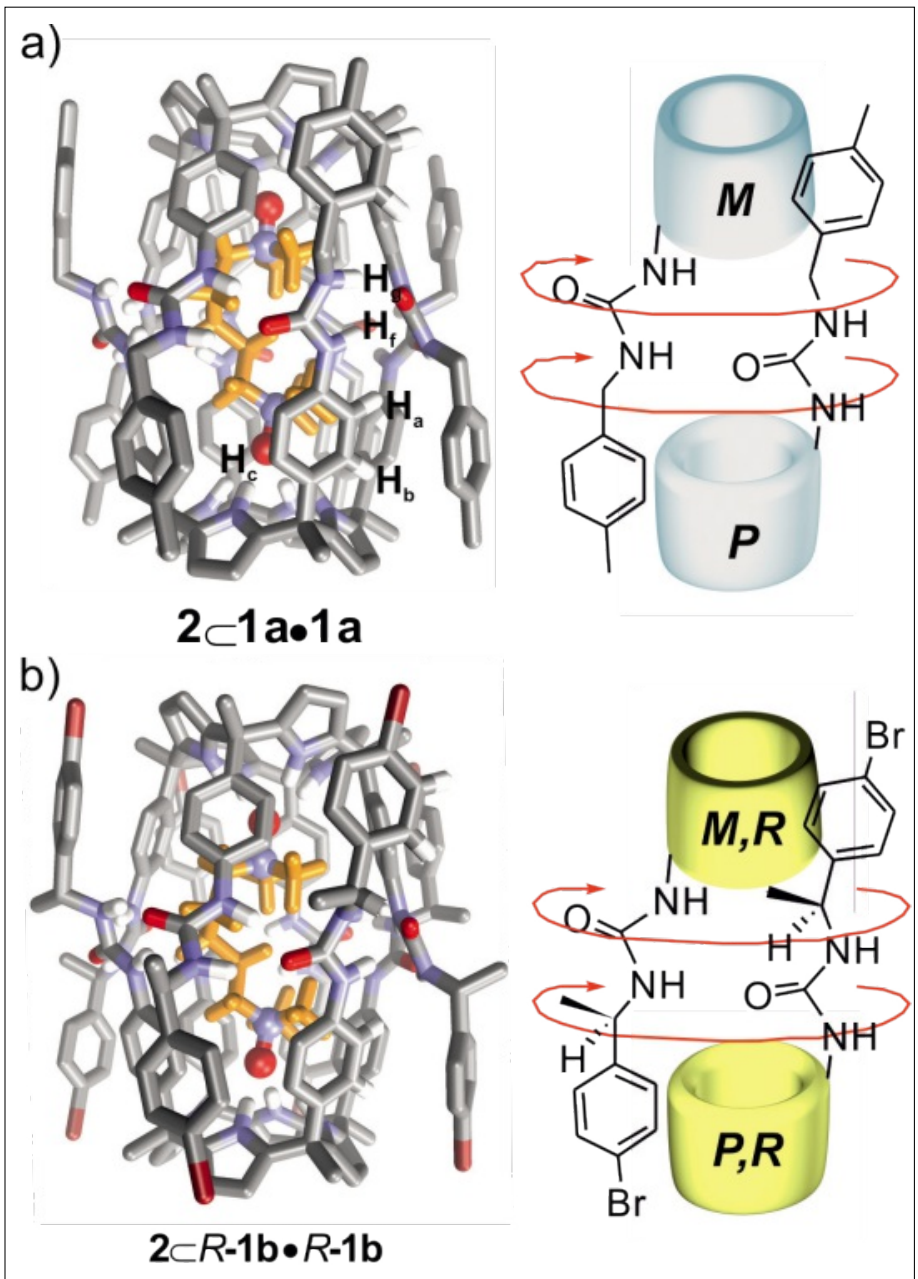

Fig. 5. Energyminimized MM3 structures of the homodimer 2 $\subset \mathbf{1 a} \bullet \mathbf{1 a}(\mathrm{a})$ and the homodimer $2 \subset R-1 \mathrm{~b} \cdot R-1 \mathrm{~b}$ (b). The corresponding schematic representations of the complexes are also depicted with the assignments of the sense of rotation of the urea groups in each hemisphere. Non-polar hydrogen atoms of the host are omitted for clarity. verted into $M$ and vice versa). Most likely, the cycloenantiomerization process of the halves was responsible for the chemical exchange process observed for the diastereotopic methyl groups. The diastereotopic methyl groups of bound 2 could also be involved in a chemical exchange process by the tumbling motion of the guest inside the capsule. However, we considered that the reduced space available inside the $\mathbf{1 a} \cdot \mathbf{1 a}$ capsule together with the coincidence in the values of the energy barriers for the cycloenantiomerization process of the two halves and the exchange of the diastereotopic methyl groups constitute strong evidence to rule out this latter possibility.

The enantiopure chiral tetraurea-calix[4]pyrrole $R$-1b differs from the nonchiral tetraurea 1a in the substitution of one of its hydrogen atoms at its four benzylic positions by a methyl group. The structure contains four stereogenic carbon centers with the same absolute configuration $(R)$. The enantiomerically pure chiral tetraurea $R \mathbf{- 1 b}$ and guest $\mathbf{2}$ were dissolved in $\mathrm{CDCl}_{3}$ solution in a 2:1 molar ratio. The analysis of the resulting solution using ${ }^{1} \mathrm{H}$ NMR spectroscopy revealed the diagnostic proton signals expected for their quantitative assembly into the dimeric capsule $\mathbf{2} \subset R-\mathbf{1 b} \bullet R-\mathbf{1 b}$ (Fig. 6b). We have mentioned above that the senses of rotation of the four urea groups in the two halves of stituent $\left(\mathrm{H}_{\mathrm{a}}, \mathrm{H}_{\mathrm{a}}{ }^{\prime}, \mathrm{H}_{\mathrm{b}}\right.$ and $\mathrm{H}_{\mathrm{b}}{ }^{\prime}$, Fig. 6a). The observed desymmetrization of the mesoaromatic protons also required that the rotation of the $\mathrm{C}_{\text {meso }}$-phenyl bond was slow on the ${ }^{1} \mathrm{H}$ NMR chemical shift timescale. Not surprisingly, the benzylic protons $\left(\mathrm{H}_{\mathrm{h}} / \mathrm{H}_{\mathrm{h}}{ }^{\prime}\right.$, Fig. 3) of 1a•1a also appeared as diastereotopic signals (Fig. 6a).

An EXSY experiment performed on a $\mathrm{CDCl}_{3}$ solution of $\mathbf{2} \subset \mathbf{1 a} \cdot \mathbf{1 a}$ evidenced the existence of a chemical exchange process between the two singlets of the diastereotopic methyl groups for the encapsulated 2 (Fig. 6c). Integration of the diagonal and chemical exchange cross-peaks for the methyl proton signals provided an energy barrier of $16.0 \mathrm{kcal} / \mathrm{mol}$ for the exchange process. The calculated energy barrier coincided with the one determined for the change in the unidirectional sense of rotation of the urea groups $(16.6 \mathrm{kcal} / \mathrm{mol})$ using the integrals of the diagonal and the cross-peaks of the diastereotopic aromatic protons ortho to the urea groups in the meso-phenyl substituents. [26] In homodimeric capsular assemblies of non-chiral building blocks (the same tetraurea-calix[4]pyrrole forms the two hemispheres), the change in the sense of rotation of the urea groups defines exactly the same molecule and simply interconverts one hemisphere into its enantiomer (the $P$ hemisphere is con-

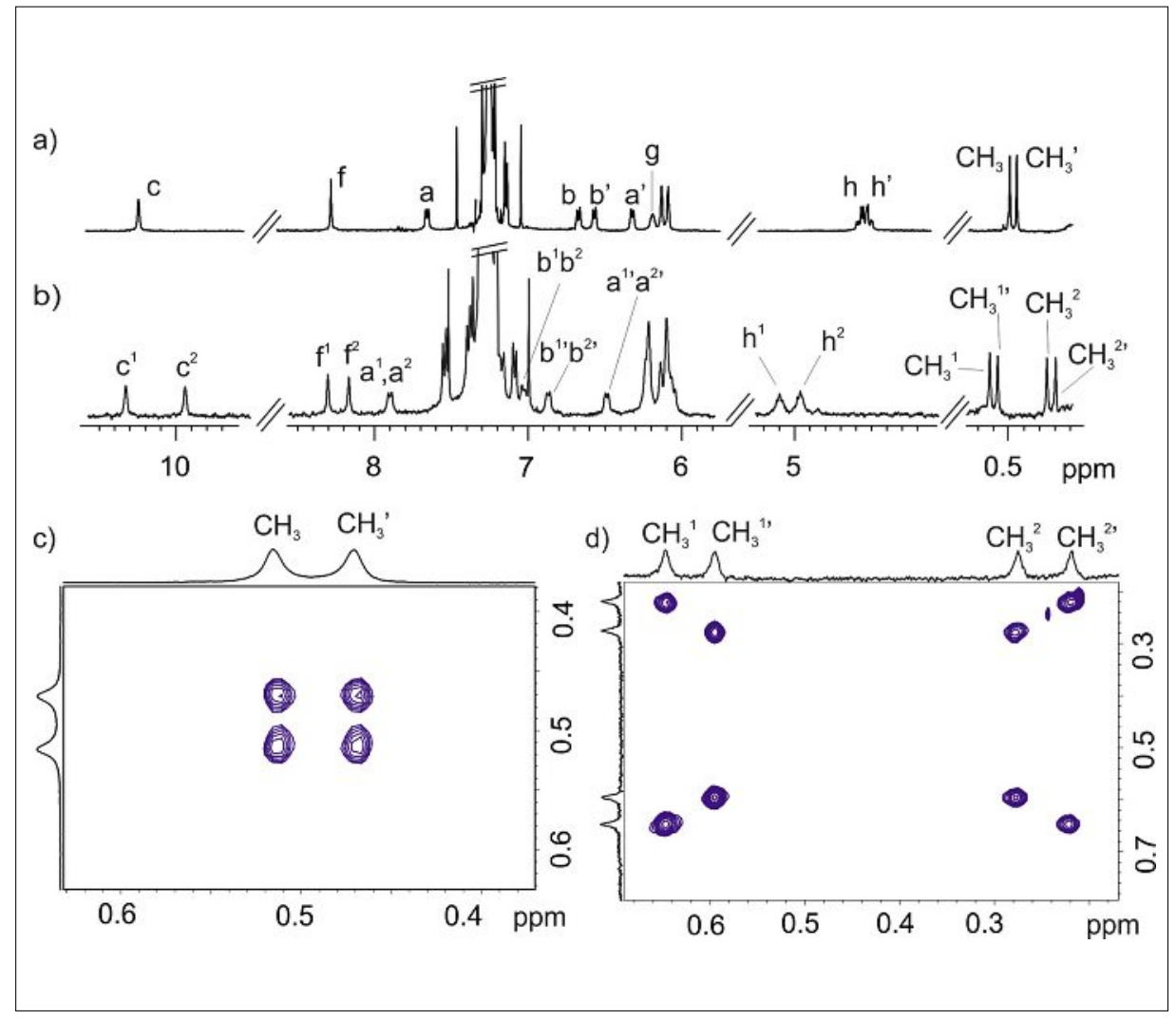

Fig. 6. Selected regions of the ${ }^{1} \mathrm{H}$ NMR spectra in $\mathrm{CDCl}_{3}$ of: a) $P-\mathbf{1 a} \bullet M R-\mathbf{1 b}$ homocapsule and $\mathrm{b}$ ) $\mathbf{2} \subset R-\mathbf{1 b} \cdot R-\mathbf{1 b}$ homocapsule. Selected upfield region of the EXSY experiments (mixing time 300 ms) of: c) $\mathbf{2} \subset \mathbf{1 a} \cdot \mathbf{1 a}$ homocapsule and d) $\mathbf{2} \subset R-\mathbf{1 b} \cdot R-\mathbf{1 b}$ homocapsule. Primed letters indicate diastereotopic protons, 1 and 2 superscripts indicate methyl groups in different hemispheres. 
the capsule must be complementary. Thus, the combination of this element of asymmetry with the existence of one stereogenic carbon in each urea arm of $R \mathbf{- 1 b}$ determined a diastereomeric relationship ( $P R$ and $M R$ ) between the two hemispheres of the dimer. Each hemisphere was chiral and featured $C_{4}$ symmetry. The entire enantiomerically pure dimeric assembly $2 \subset P R$ 1b•MR-1b also showed $C_{4}$ symmetry. Because the two hemispheres have a diastereomeric relationship, they are distinct: chemically non-equivalent. Consequently, their proton signals, as well as those of the included $N$-methyl groups of the guest might have different chemicals shifts. The reduction in symmetry described for the capsule $\mathbf{2} \subset P R-\mathbf{1 b} \bullet M R-\mathbf{1 b}$ compared to the previous homodimeric $\mathbf{1 a} \bullet \mathbf{1 a}$ capsule, was reflected in a larger magnetic asymmetry of its inner space and the concomitant increase in the number of proton signals of the encapsulated guest. We observed two sets of two diastereotopic singlets for the methyl groups of the encapsulated $\mathbf{2}$ in the ${ }^{1} \mathrm{H}$ NMR spectrum of $\mathbf{2} \subset P R-\mathbf{1 b} \bullet M R-\mathbf{1 b}$. Each $N$-oxide knob is included in a distinct chiral hemisphere and yielded one set of two diastereotopic singlets. The existence of chemically non-equivalent hemispheres in the $\mathbf{2} \subset P R-\mathbf{1 b} \bullet M R-\mathbf{1 b}$ assembly was also substantiated by the observation of two separated proton signals for the pyrrole $\mathrm{NHs}$ (each integrating by four protons). We commented above that the combination of the same tetraurea monomer in $\mathbf{2} \subset \mathbf{1 a \bullet 1 a}$ defines a cycloenantiomerization process of the halves ( $M$ became $P$ and vice versa) through the change in the unidirectional orientation of the urea groups and the concomitant existence of a chemical exchange process between diastereotopic methyl groups of bound 2 . In striking contrast, the analogous change of orientation of the urea groups in the homocapsule $2 \subset P R$ $\mathbf{1 b} \cdot M R-\mathbf{1 b}$ (having chemically non-equivalent hemispheres) interconverted the two diastereomeric halves $(M R$ into $P R$ and vice versa) but without inducing their enantiomerization. Accordingly, the EXSY chemical exchange pattern for the methyl signals of $\mathbf{2}$ encapsulated in $P R-\mathbf{1 b} \bullet M R$ 1b showed exchange cross-peaks only between pairs of methyl groups included in distinct hemispheres (Fig. 6d).

\section{Magnetic Asymmetries in the Inner Space of Chiral Heterodimeric Capsular Assemblies: $1 \mathrm{a} \bullet \boldsymbol{R}-\mathbf{1 b}$ and 1a•1c}

We previously reported that the assembly of 1a with $R$-1b induced by encapsulating one molecule of 4,4'-dipyridyl $N, N^{\prime}$-dioxide 3 yielded exclusively the heterocapsule aggregate $\mathbf{3} \subset P-\mathbf{1 a} \bullet M R-\mathbf{1 b} .{ }^{[24]}$
We demonstrated that upon heterodimerization, the counterclockwise $(M)$ sense of rotation of the urea groups in tetraureacalix[4]pyrrole $R-\mathbf{1 b}$ was energetically favored. This result derived from an efficient transfer of chirality of the stereogenic center to the cyclochiral conformer. Consequently, the assembly of tetraurea 1a in a dimeric capsule with $R-\mathbf{1 b}$ forced the former to adopt the $P$ conformational cyclochirality. ${ }^{[24]}$ Theoretical calculations indicated that the diastereomeric heterocapsule $\mathbf{3} \subset M-\mathbf{1 a} \cdot P R-\mathbf{1 b}$ was significantly higher in energy compared to $\mathbf{3} \subset P-\mathbf{1 a} \bullet M R$ 1b. We concluded that the heterodimeric chiral assembly $\mathbf{3} \subset P \mathbf{- 1 a} \bullet M R \mathbf{- 1 b}$ did not experience a change in the unidirectional orientation of their urea groups because the resulting dimer $\mathbf{3} \subset M-\mathbf{1 a} \bullet P R \mathbf{- 1} \mathbf{b}$ was thermodynamically unstable.

An equimolar mixture of the non-chiral tetraurea-calix[4]pyrrole 1a and the enantiomerically pure tetraurea-calix[4]pyrrole $R-\mathbf{1 b}$ could produce a maximum of four different capsular assemblies encapsulating one molecule of 2 : two homodimeric capsules $(\mathbf{2} \subset \mathbf{1 a} \cdot \mathbf{1 a}$ and $\mathbf{2} \subset R-\mathbf{1 b} \bullet R-\mathbf{1 b})$ and two heterodimeric assemblies $(\mathbf{2} \subset(M-\mathbf{1 a} \bullet P R$ 1b) and $\mathbf{2} \subset(P-\mathbf{1 a} \bullet M R-\mathbf{1 b}))$ that are chiral diastereoisomers. Experimentally, the ${ }^{1} \mathrm{H}$ NMR spectrum of the mixture showed the diagnostic proton signals for the exclusive assembly of only one of the two hetero- dimers (Fig. 7a). By analogy to the results commented above, we assigned the absolute configuration $\mathbf{2} \subset P-\mathbf{1 a} \bullet M R-\mathbf{1 b}$ to the exclusively produced heterodimer in the present work (Fig. 8).

The ${ }^{1} \mathrm{H}$ NMR spectrum of a $\mathrm{CDCl}_{3}$ solution containing the $\mathbf{2} \subset P-\mathbf{1 a} \cdot M R \mathbf{- 1} \mathbf{b}$ heterodimer showed that the methyl protons of the encapsulated guest $\mathbf{2}$ appeared as two sets of two separated singlets (Fig. 7a). This splitting pattern was in agreement with the existence of two distinct chiral hemispheres. Each hemisphere included one dimethyl- $N$-oxide terminal group of the encapsulated guest 2 . The methyl groups of the two chemically nonequivalent terminal $\mathrm{N}$-oxide groups of bound 2 also became diastereotopic due to the chiral nature of the hemispheres and resonated as two separated signals. A transverse T-ROESY[27] experiment performed on the above solution showed the total absence of cross-peaks between the methyl proton signals of the encapsulated $\mathrm{N}$-oxide 2. This fact evidenced the lack of chemical exchange processes involving the methyl groups and supported the existence of a unique and preferred sense of orientation of the urea groups in the heterodimeric chiral assembly $\mathbf{2} \subset P-\mathbf{1 a} \bullet M R-\mathbf{1 b}$ (Fig. 7c).

The non-chiral tetraurea-calix[4]pyrrole $1 \mathbf{a}$ (1 equiv.) and the non-chiral bisloop calix[4]pyrrole derivative 1c (1.2 equiv.)

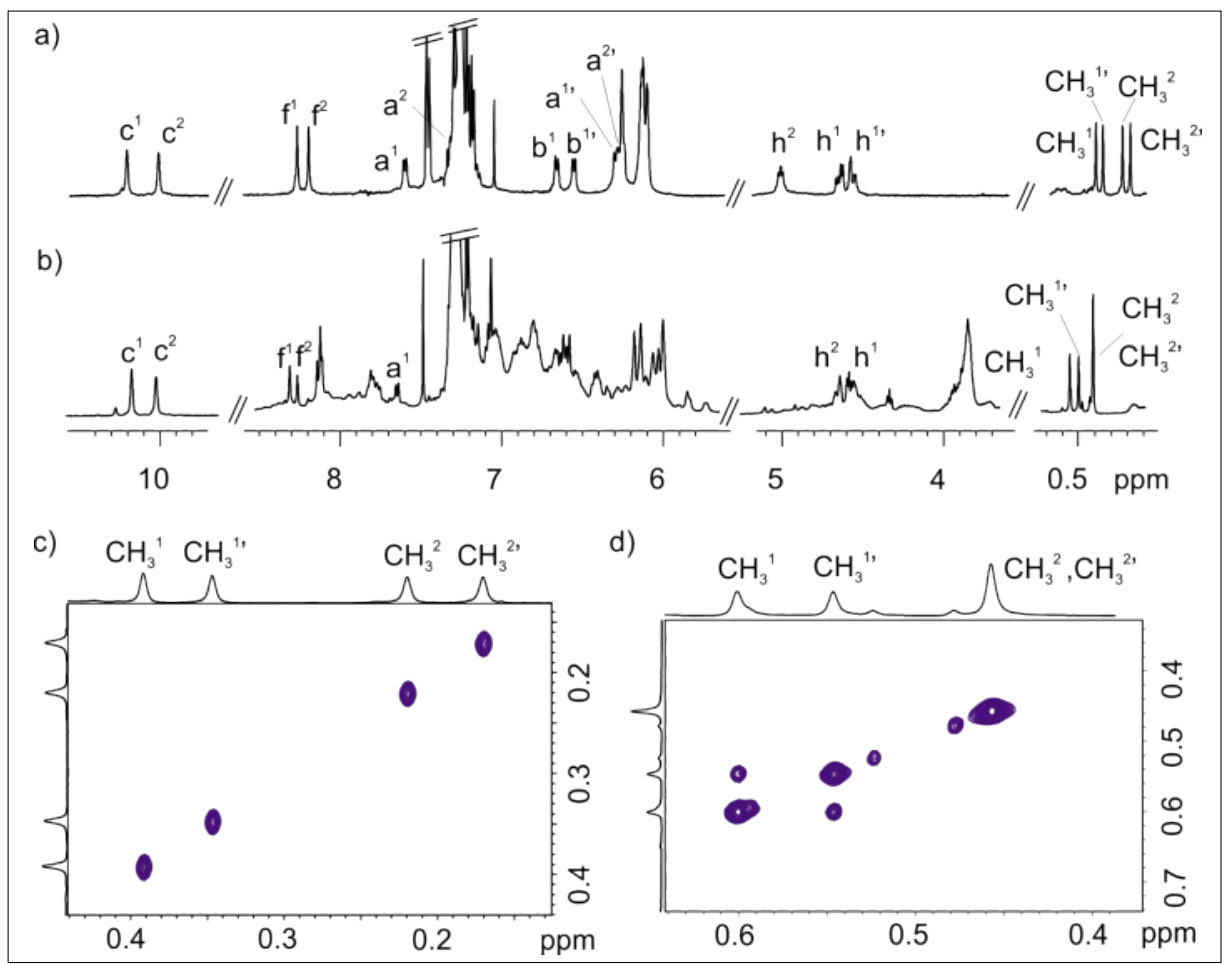

Fig. 7. Selected regions of the ${ }^{1} \mathrm{H}$ NMR spectra in $\mathrm{CDCl}_{3}$ of: a) $\mathbf{2} \subset \mathbf{1 a} \cdot \mathbf{R}-\mathbf{1} \mathbf{b}$ heterocapsule and b) $2 \subset \mathbf{1 a} \cdot 1 \mathrm{c}$ heterocapsule. Selected upfield region of: c) the transverse T-ROESY experiments of $\mathbf{2} \subset \mathbf{1 a} \cdot R-\mathbf{1 b}$ heterocapsule and d) the EXSY experiment of $\mathbf{2} \subset \mathbf{1 a} \mathbf{1 c}$ heterocapsule, the small dou-

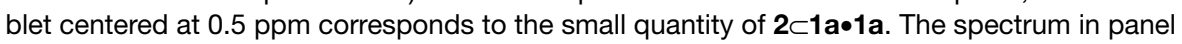
c was acquired using the transverse T-ROESY pulse program in order to remove artifact crosspeaks (HOHAHA). Primed letters indicate diastereotopic protons, 1 and 2 superscripts indicate different hemispheres. 


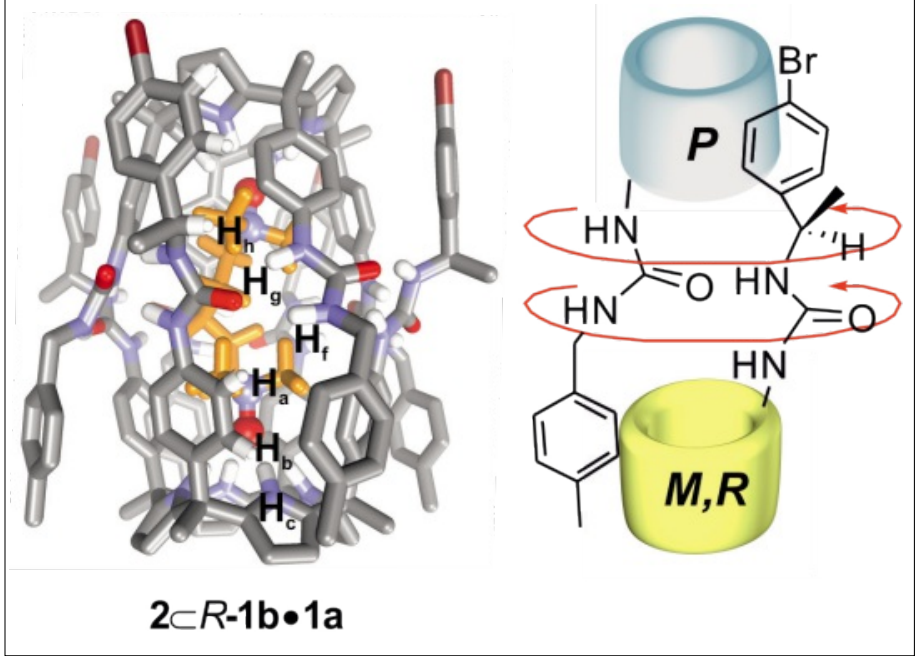

Fig. 8. Energy minimized MM3 structure of heterodimer $2 \subset P-1 a \cdot M R-1 b$ and its corresponding schematic representation with indication of the sense of rotation of the urea groups in each hemisphere. Non-polar hydrogen atoms of the host are omitted for clarity. assembled almost quantitatively in $\mathrm{CDCl}_{3}$ solution in the presence of 2 ( 1 equiv.). The mixture yielded the heterodimeric capsule $\mathbf{2} \subset \mathbf{1 a \bullet 1 c}$ as the major assembly. A careful analysis of the resulting solution using ${ }^{1} \mathrm{H}$ NMR spectroscopy revealed the presence of traces of the homodimer $\mathbf{2} \subset \mathbf{1 a} \bullet \mathbf{1 a}$ (Fig. 7b). None of the components of the

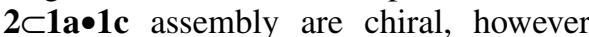
the required complementary between the senses of rotation of the urea groups of the two hemispheres forced the two halves to adopt cyclochiral conformations. Again, the unidirectional orientation of the urea groups in the two capsules was kinetically stable on the ${ }^{1} \mathrm{H}$ NMR timescale. This produced the existence of the $\mathbf{2} \subset \mathbf{1 a} \bullet \mathbf{1 c}$ capsule in solution as a mixture of two enantiomers, $\mathbf{2} \subset M-\mathbf{1 a} \bullet P-\mathbf{1 c}$ and $\mathbf{2} \subset P-\mathbf{1 a} \bullet M$ 1c, which interconverted by changing the unidirectional sense of rotation of their urea groups (Fig. 9). In each enantiomeric capsule the two halves are chemically nonequivalent and conformationally chiral. In short, the methyl groups of encapsulated 2 are expected to resonate as four separated singlets.

In complete agreement with these symmetry considerations, the ${ }^{1} \mathrm{H}$ NMR spectrum of the racemic heterocapsule 2 $\subset 1 \mathbf{1 a} \cdot 1 \mathrm{c}$ in $\mathrm{CDCl}_{3}$ solution showed two separate proton signals for the pyrrole $\mathrm{NHs}$ indicative of two distinct hemispheres (Fig. 7b). However, only three different signals were detected for the methyl protons of encapsulated 2. Two singlets integrated for three protons and one for six protons. Most likely, two of the singlets of the four methyl groups in bound $\mathbf{2}$ resonate at the same chemical shift. A 2D EXSY experiment revealed the existence of chemical exchange cross-peaks between signals that were assigned to the diastereotopic methyl groups of encapsulated 2 (Fig. 7d). The energy barrier calculated for the exchange process was in agreement with the energy required for the inversion of the unidirectional orientation of the urea groups $(17.3 \mathrm{kcal} / \mathrm{mol})$. Taken together, these results suggested that the chemical exchange between diastereotopic methyl protons was caused by the inversion of the sense of rotation of the capsule's urea belt. Possibly and as already commented above, the tumbling motion of encapsulated $\mathbf{2}$ is not allowed owing to geometric/size con-

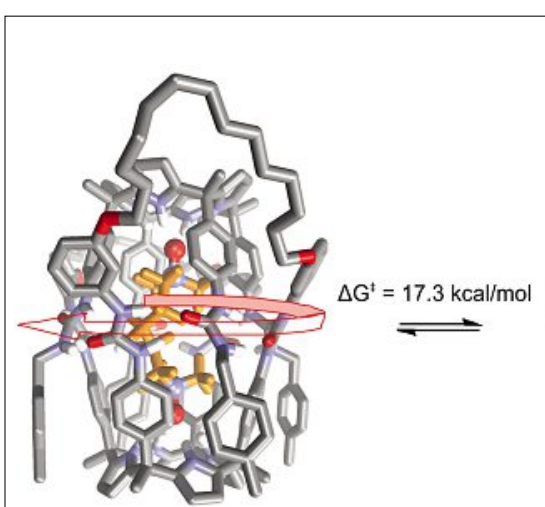

2 $\subset M-1 \mathrm{a} \bullet P-1 \mathrm{c}$

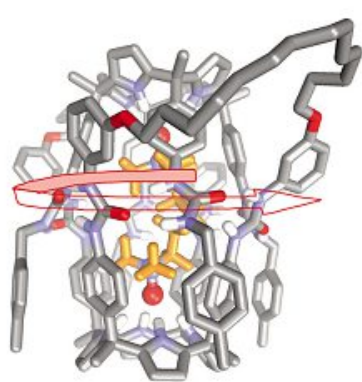

$2 \subset P-1 a \cdot M-1 c$
Fig. 9. Equilibrium between the two enantiomeric capsules 2ᄃM-1a॰P-1c and 2ᄃP-1a•M-1c. The red arrows indicate the unidirectional sense of rotation of the belt of hydrogen bonded ureas assigned by looking from the top of the bisloop component. The inversion of the unidirectional sense of rotation of the urea groups interconverts the two enantiomers. straints of the container. This hypothesis was substantiated experimentally by the lack of exchange cross-peaks between the methyl groups encapsulated in distinct hemispheres.

\section{Conclusions}

We have assembled and studied a series of dimeric molecular capsules based on tetraurea-calix[4]pyrrole derivatives. All capsules encapsulated one molecule of the bis- $N$-oxide 2 . We observed capsule's dependent splitting pattern for the $N$-methyl proton signals of encapsulated 2 (from 2 to 4 separate singlets) that reflected the differences in magnetic asymmetry of their interiors. We rationalized the observed splitting patterns based on the symmetry and the supramolecular chirality properties of the capsular assemblies, as well as of their components. The explanations of the observed number of signals for the methyl groups of bound $\mathbf{2}$ are also supported by the results obtained in the corresponding 2D EXSY/ROESY NMR experiments. The methyl groups of encapsulated $\mathbf{2}$ were involved in chemical exchange processes caused by the fast inversion, on the EXSY/ ROESY timescale, of the sense of rotation of the belt of hydrogen-bonded urea groups that stabilized the capsules. In contrast, the unidirectional orientation of the urea groups was kinetically stable on the chemical shift timescale and was responsible for the existence in solution of the capsule's hemispheres as complementary cyclochiral conformers. The inclusion of the dimethyl- $N$-oxide knobs of $\mathbf{2}$ in the chiral components of the capsules rendered the methyl groups of the former diastereotopic. The ${ }^{1} \mathrm{H}$ NMR spectra of capsules featuring enantiomeric hemispheres $(\mathbf{1 a} \cdot \mathbf{1 a})$ displayed one set of two singlets for the methyl protons of encapsulated 2. On the other hand, the ${ }^{1} \mathrm{H}$ NMR spectra of capsules with diastereomeric $(M R-\mathbf{1 b} \cdot P R$ 1b) or chemically non-equivalent hemispheres $(\mathbf{1 a} \cdot R-\mathbf{1 b}$ and $\mathbf{1 a} \bullet \mathbf{1 c})$ showed two sets of two singlets for the methyl groups of encapsulated 2. The obtained results ruled out the existence of a tumbling motion of encapsulated 2. Conversely, the $C_{4}$ symmetry displayed by the hemispheres of the capsules suggested a fast spinning process on the chemical shift timescale of encapsulated 2.

\section{Supplementary Data}

Experimental details, synthesis and characterization of calyx[4]pyrrole 1c and details on the calculations of the energy barriers of the chemical exchange processes detected by $2 \mathrm{D}$ EXSY experiments. 


\section{Acknowledgements}

The authors thank Gobierno de España MINECO (projects CTQ2014-56295-R, CTQ2014-52974-REDC and Severo Ochoa Excellence Accreditation 2014-2018 SEV2013-0319), FEDER funds (project CTQ201456295-R) and the ICIQ Foundation for funding.

Received: September 10, 2015

[1] L. Zhang, L. Qin, X. Wang, H. Cao, M. Liu, Adv. Mater. 2014, 26, 6959.

[2] J. Crassous, Chem. Soc. Rev. 2009, 38, 830.

[3] A. Scarso, J. Rebek, in 'Supramolecular Chirality', Eds. M. Crego-Calama, D. N Reinhoudt, Springer-Verlag: Berlin, Heidelberg, 2006, vol. 265, p. 312 .

[4] M. A. Mateos-Timoneda, M. Crego-Calama, D. N. Reinhoudt, Chem. Soc. Rev. 2004, 33, 363.

[5] M. Liu, L. Zhang, T. Wang, Chem. Rev. 2015, $115,7304$.

[6] A. Szumna, Chem. Soc. Rev. 2010, 39, 4274

[7] M. Grajda, M. Wierzbicki, P. Cmoch, A. Szumna, J. Org. Chem. 2013, 78, 11597.
[8] A. V. Davis, D. Fiedler, M. Ziegler, A. Terpin, K. N. Raymond, J. Am. Chem. Soc. 2007, 129, 15354.

[9] L. J. Prins, J. Huskens, F. de Jong, P. Timmerman, D. N. Reinhoudt, Nature 1999, 398, 498.

[10] C. X. Lin, Y. G. Ke, Z. Li, J. H. Wang, Y. Liu, H. Yan, Nano Lett. 2009, 9, 433.

[11] T. Imura, N. Ohta, K. Inoue, N. Yagi, H. Negishi, H. Yanagishita, D. Kitamoto, Chem.Eur. J. 2006, 12, 2434.

[12] Y. Tsunoda, K. Fukuta, T. Imamura, R. Sekiya, T. Furuyama, N. Kobayashi, T. Haino, Angew. Chem., Int. Ed. 2014, 53, 7243.

[13] P. L. Arnold, J. C. Buffet, R. Blaudeck, S. Sujecki, C. Wilson, Chem.-Eur. J. 2009, 15, 8241.

[14] C. G. Claessens, T. Torres, J. Am. Chem. Soc. 2002, 124, 14522.

[15] M. Yoshizawa, M. Tamura, M. Fujita, Angew. Chem. Int. Ed. 2007, 46, 3874.

[16] M. Yoshizawa, M. Fujita, Pure Appl. Chem. 2005, 77, 1107.

[17] M. Yoshizawa, J. K. Klosterman, M. Fujita, Angew. Chem. Int. Ed. 2009, 48, 3418.
[18] R. K. Castellano, B. H. Kim, J. Rebek, J. Am. Chem. Soc. 1997, 119, 12671.

[19] B. Kuberski, A. Szumna, Chem. Commun. 2009, 1959.

[20] A. Szumna, Chem.-Eur. J. 2009, 15, 12381.

[21] T. Amaya, J. Rebek, J. Am. Chem. Soc. 2004, $126,6216$.

[22] M. P. Schramm, P. Restorp, F. Zelder, J. Rebek, J. Am. Chem. Soc. 2008, 130, 2450.

[23] P. Ballester, G. Gil-Ramirez, Proc. Natl. Acad. Sci. USA 2009, 106, 10455.

[24] M. Chas, G. Gil-Ramirez, E. C. Escudero-Adan, J. Benet-Buchholz, P. Ballester, Org. Lett. 2010, $12,1740$.

[25] M. Espelt, P. Ballester, Org. Lett. 2012, 14, 5708.

[26] G. Gil-Ramirez, M. Chas, P. Ballester, J. Am. Chem. Soc. 2010, 132, 2520.

[27] A. Bax, D. G. Davis, J. Magn. Reson. 1985, 63, 207. 